\title{
Incorporating Image Priors with Deep Convolutional Neural Networks for Image Super-Resolution
}

\author{
Yudong Liang, Jinjun Wang*, Sanping Zhou, Yihong Gong, Nanning Zheng \\ Institute of Artificial Intelligence and Robotics, Xi'an Jiaotong University, 28 West \\ Xianning Road, Xi'an, Shaanxi, China, 710049
}

\begin{abstract}
Deep convolutional neural network has been applied for single image superresolution problem and demonstrated state-of-the-art quality. This paper presents several prior information that could be utilized during the training process of the deep convolutional neural network. The first type of prior focuses on edges and texture restoration in the output, and the second type of prior utilizes multiple upscaling factors to consider the structure recurrence across different scales. As demonstrated by our experimental results, the proposed framework could significantly accelerate the training speed for more than ten times and at the same time lead to better image quality. The generated super-resolution image achieves visually sharper and more pleasant restoration as well as superior objectively evaluation results compared to state-of-the-art methods.

Keywords: super-resolution, Deep convolutional neural network, image gradient priors, multi-task learning
\end{abstract}

\section{Introduction}

Image super-resolution (SR) aims to overcome the resolution limitation of the sensors and restores high resolution (HR) images from low resolution(LR) inputs with information complementing. Image super-resolution is a classic yet 5 an inherently ill-possed problem as different HR images can be degenerated into

\footnotetext{
${ }^{*}$ Corresponding author
}

Preprint submitted to Journal of ${ }^{A} T_{E} X$ Templates

January 12, 2016

(C) 2016. This manuscript version is made available under the Elsevier user license http://www.elsevier.com/open-access/userlicense/1.0/ 
the same LR image. To handle the under-determined nature of the problem, various prior knowledge have been utilized. For instance, both the interpolation methods [1] and the reconstruction methods [2, 3] assume that pixels change only gradually in a local area, such that the former utilizes local smooth functions for interpolation, and the latter uses coherence constraints in the optimization problems for reconstruction.

In another evolving and promising line of methodology, the example based approach [4, 5], the recurrence priors between LR and HR image examples are modeled by learning a mapping from internal/external examples [4, 6] or from combined sources [7. In the pioneer work by Freeman et al 4, the co-occurrence prior between LR and HR image patches were integrated into an Markov Random Field model [4] to restore locally coherent HR images. Yang [5, 8] proposed to combine the learned dictionary with sparse coding coefficients estimated among signals to restore HR image patches. Zhang [9] further explored nonlocal multi-scale similarity to get better results. Some researchers have also attempted analyzing the blurring kernel that causes the degeneration between HR examples and LR examples. For example, Michaeli [10] estimated the optimal blur kernel by the inherent recurrence property of small image patches. Timofte [11] and Yang [12] grouped the feature spaces of image patches into nu25 merous subspaces which indirectly distinguished the blur model and got better performance. Yang [13] systematically analyzed the impact of the blur kernel and made a benchmark comparison for several state of art methods.

In fact, the structure recurrence among examples and the low degree of freedom in local image structures 9 make it possible for a feasible learning of a mapping, such that the problem becomes learning a regression model. The promising performance demonstrated by deep-learning methods in recent years has inspired researchers to attempt deep models for optimal HR restoration. For instance, Dong [14] proposed a deep convolutional neural network for image super-resolution. It directly learned end-to-end mappings from LR to HR images which achieved state-of-the-art restoration quality. However, the parameters in a deep model are usually highly free, and learning the purely end to end 


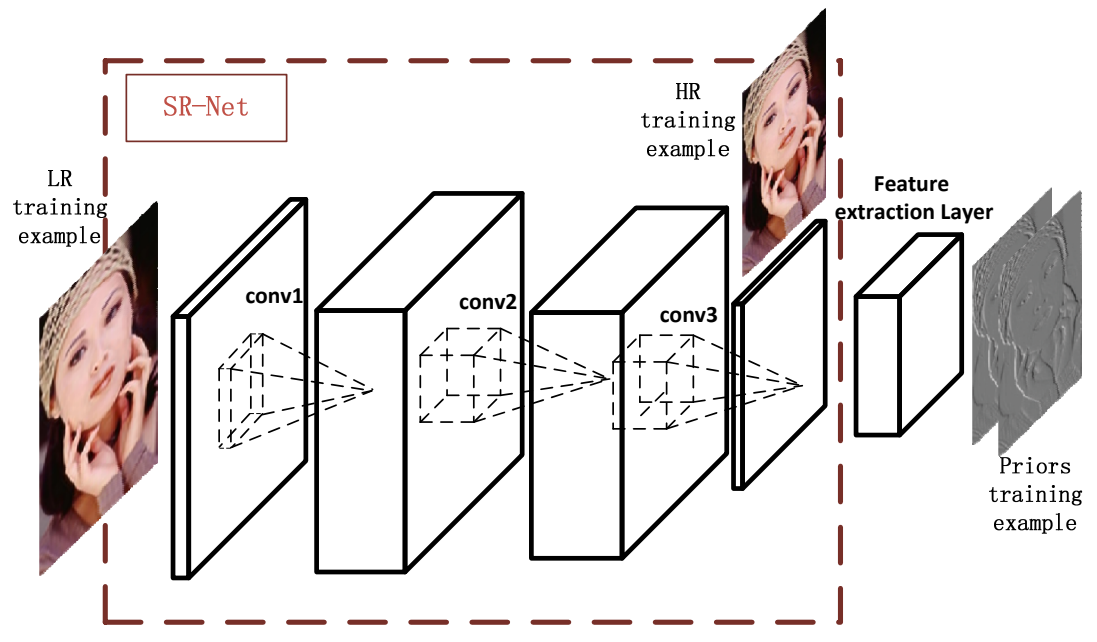

Figure 1: The proposed Convolutional network framework with gradient and texture priors modeled,denoted as SRCNN-Pr

mappings would often fall into local minimum, especially when only limited training images are available as that in the SR problem. On the other side, as demonstrated in 14, 15, it needs days for training given large amount of

40 training samples. Thus, how to incorporate useful prior to constrain or regulate the learning process of a deep model is a critical issue both for speeding up the training and improving image quality in the SR task. Lee [16] argued that supervised constraints would contribute to learning discriminative features and accelerating the convergence process. Besides, Dong [14, 15] emphasized that large sub-image examples were important.

In this paper, we incorporated the image priors such as image gradients into constructing deep convolutional neural network and further extend the deep convolutional network with multi-scale information regulated by multi-task learning. As in Fig 1, fixed feature extraction layers were concatenated with the SR-Net [14] and feature of image gradients were obtained. Thus, the training process could pay special attention to the image gradients. A loss function was optimized together with mapping from LR to HR sub-images. The consistency of features extracted between reconstructed images and original images was 
propagated to supervise the training of mapping from LR to HR sub-images. Besides, we naturally extend the framework to support multi-task learning in order to simultaneously train deep convolutional neural network with different magnification factors, as illustrated in Fig.2. The local structures in natural images usually tend to reoccur across different scales, and therefore the multiscale information could be shared in all the tasks. With weight sharing, less parameters are needed and better results are achievable. Finally, we combined the two priors to give a superior results with a faster training convergency rate.

The rest of this paper is organized as follows. In section 2, we describe the proposed framework consisting of a deep convolutional neural network with image gradient priors. We denote the framework as SRCNN-Pr. In section 3, we present our multi-task learning deep convolutional network which was further combined with image multi-scale magnification priors, denoted as SRCNNMultitask-Pr. Experimental results and comparisons with state-of-the-art methods are provided in Section 4, and we conclude the paper in Section 5.

\section{Deep convolutional neural network framework with priors and multi-task learning for multi-scale image SR}

A LR image $I^{L}$ can be degenerated from the corresponding HR image $I^{H}$ with the following blurring and downsampling process as Eq.(1).

$$
I^{L}=\left(I^{H} \bigotimes G\right) \downarrow_{\frac{1}{s}}
$$

$G$ denotes a blur kernel and different images may relate to different kernels.

$\downarrow_{\frac{1}{s}}$ is the downsample process with a scale factor $S$. LR sub-images $\left\{\mathbf{I}^{l}\right\}$ and HR sub-images $\left\{\mathbf{I}^{h}\right\}$ were extracted from LR and HR images respectively to form the training set $\left\{\mathbf{I}^{l}, \mathbf{I}^{h}\right\}$. In fact, different training HR examples were downsized by bicubic interpolation to generate LR-HR image pairs for both training and evaluation as 14. It mimicked that different training examples were blurred with different kernels, thus learning a good mapping from LR examples to HR examples should handle these complex situations. With deep 
convolutional neural networks, the end to end mapping must be well trained with sufficient number of training examples. As in Fig 1, the first three Layers of deep convolutional neural network have been applied to restore HR Images in test phase which has the same structure as Dong's CNN network 14. The images or feature maps $\left\{X_{j}\right\}$ of the $j$ th convolutional layer are convolved by a set of filters $W$. And biases $\mathbf{B}$ of the filters are always added as Eq.(2).

$$
Y_{j}=W * X_{j}+B
$$

The first two convolutional layers are followed by an rectified linear units(RELU). Thus it becomes Eq.(3).

$$
Y_{j}=\max \left(0, W * X_{j}+B\right)
$$

The model parameters are denoted as $\theta=\left\{W_{i}, B_{i}\right\}$. The reconstruction loss $\mathrm{Loss}_{r}$ is penalized as Eq.(4).

$$
\operatorname{Loss}_{r}=\operatorname{loss}\left(Y_{3}, \mathbf{I}^{h}\right)=\left\|Y_{3}-\mathbf{I}^{h}\right\|^{2}
$$

\subsection{Deep convolutional neural network with gradients priors}

Although it already got great results with just a few convolutional layers, the artifacts still existed in some edge and rich texture areas. Besides, the training time was too long and Dong [14] declared the net was far from convergence. If a correctly restored image is built, edges(related to the image gradients) and texture should be especially authentic. At the same time, if authentic image gradients or edges and texture areas have been reconstructed, we would achieve visually appealing SR results. Thus, after the third convolutional layer, a feature extraction layer was followed to pay special attention to image gradients and textures. In fact, the domain priors can be generic and are not limited to image gradients, image texture and other features describing the image can be further explored. A loss function supervised the restored image gradients to be authentic as Eq. (5).

$$
\operatorname{Loss}_{P r}=\operatorname{loss}\left(f_{P r}\left(Y_{3}, \theta\right), f_{P r}\left(\mathbf{I}^{h}\right)\right)
$$


$f_{P r}$ means incorporating priors, such as extracting image gradient information. Overall, the reconstruction error is consisted of the reconstruction error and feature consistency measure as Eq. (6).

$$
\text { Loss }=\operatorname{Loss}_{r}+\alpha \cdot \operatorname{Loss}_{P r}
$$

The balance parameter $\alpha$ is a trade-off between the reconstruction objective and the respective feature consistency measure. A zero-ing training approach was applied in the training which means the balance parameter $\alpha$ would gradually decrease in the training. Commonly, when loss of the second term as Eq.(6) falls below a threshold (a hyper parameter) $\gamma$, the $\alpha$ vanishes and the second loss term no longer contributes to the gradients updating process. Alternatively, with zeroing method, a simple decay process as Eq.(7) or Eq.(8) aims to enforce the second term to vanish after certain number of iterations, where $t_{\text {epoch }}$ is the epoch step and Numepoch $_{\text {is }}$ the total number of epochs.

$$
\begin{gathered}
\alpha=\alpha_{0} \cdot\left(1-\frac{t_{\text {epoch }}}{\text { Num }_{\text {epoch }}}\right) \\
\alpha=\alpha_{0} \cdot d^{t_{\text {epoch }}}
\end{gathered}
$$

All these mean that the overall goal of HR images reconstruction is not fundamentally altered and the image gradients consistency measure acts as a type of regularization or as a guided supervised training information. These can also be interpreted as a good initialization method by utilizing the priors like image gradients. For instance, a fixed filters bank can be applied to extract features. A sobel Edge filters [17] have been used in our experiment which generate two feature maps to capture the horizontal and vertical gradient information. The information flow from the measurement loss would be propagated to the reconstruction part of deep CNN without updating the filters. The net was minimized using stochastic gradient descent with the standard back-propagation [18. In particular, the parameter is updated as Eq.(9), where m denotes for the momentum parameter with a value 0.9 .

$$
\triangle_{i+1}=m \cdot \triangle_{i}+\eta \cdot \frac{\partial \text { Loss }}{\partial \theta_{i}}, \quad \theta_{i+1}=\theta_{i}+\triangle_{i+1}
$$


As demonstrated in the section of experiments, the sharper edges and more authentic texture areas were restored and the training converging speed was largely accelerated (more than ten times).

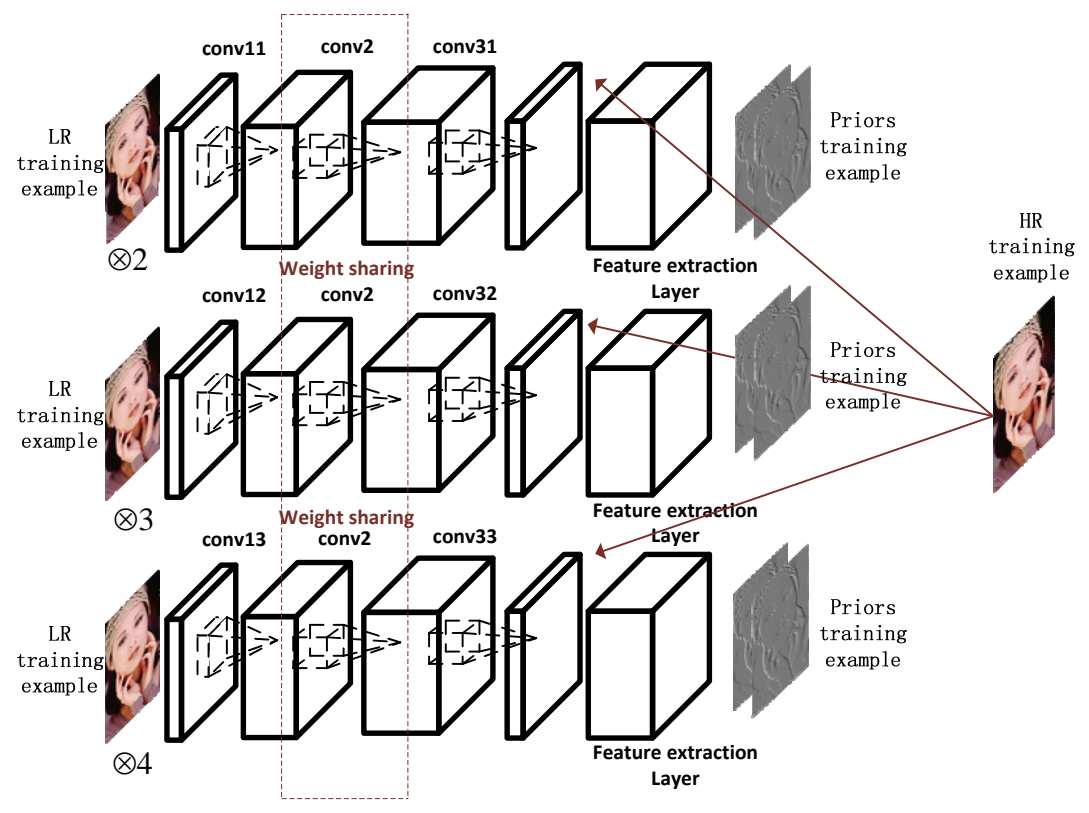

Figure 2: The proposed Convolutional network framework with gradient priors modeled, denoted as SRCNN-Pr

In the next section, a multi-task learning framework of multi-scale extension would be designed to exploit the structure recurrence of the image across the scale.

\subsection{Multi-task learning deep convolutional neural network framework for super- resolution with different magnification factors}

The local structures in natural images usually tend to reappear many times across different scales. Missing details can be estimated from its similar examples at a different scale. Different images prefer different scales for optimal representation. For instance, the striking edges and the sophisticated details tend to refer to the structure across the different scale respectively. Therefore, 
exploiting information across the different scales should contribute to the learning of mapping from LR to HR images. As in the Fig2, a multi-task learning framework has been applied to exploit the structure redundancy across different scales using a weight sharing strategy. By sharing a layer, same set of filters are applied to each path and sharing weights across different scales provides regularization to each other. Through weight sharing, information could be transferred across scales. Taking Fig 2 as an example, different training sample sets $\left\{\mathbf{I}_{\frac{1}{s}}^{l}, \mathbf{I}_{\frac{1}{s}}^{h}\right\}$ with upscaling factor $s$ were given to the three entrance of the first layers. Then the input of different training sample sets are convolved with $\operatorname{conv}_{12}, \operatorname{conv}_{13}, \operatorname{conv}_{14}$ respectively. There are three paths of the network and the second convolutional layer is shared by these three paths. Sharing a layer means same set of filters are applied to each path and different scales provide regularization to each other. The total loss of the multi-scale convolutional neural network with image priors, is demonstrated as Eq. 10].

$$
\text { Loss }=\sum_{i_{\text {scale }}} \beta_{\left(i_{\text {scale }}\right)} \cdot\left(\operatorname{Loss}_{r}^{i_{\text {scale }}}+\alpha \cdot \operatorname{Loss}_{P r}^{i_{\text {scale }}}\right)
$$
$\beta_{\left(i_{\text {scale }}\right)}$ adjusts the significance of certain path. Larger $\beta_{\left(i_{\text {scale }}\right)}$ pays more attention to certain path. we applied $\beta_{\left(i_{\text {scale }}\right)}=1$ in the experiment. The backpropagated gradients of sharing layers i can be calculated by the summation of the next layer's gradients as Eq. 11]. The significance factor $\beta_{\left(i_{\text {_scale }}\right)}$ has already been considered in $\left(\frac{\partial \text { Loss }}{\partial \theta_{i+1}}\right)^{i_{\text {scale }}}$.

$$
\frac{\partial \text { Loss }}{\partial \theta_{i}}=\sum_{i_{\text {scale }}}\left(\frac{\partial \text { Loss }}{\partial \theta_{i+1}}\right)^{i_{\text {scale }}}
$$

Less parameters needs to be learnt compared with total amount of parameters for training several deep convolutional neural network independently. Training deep convolutional neural network for super-resolution with different up-scaling factors were simultaneously accomplished. At the test time, single path of the convolutional neural network would be applied separately to do image super-resolution. For instance, the path along conv11, conv2, conv31 would 


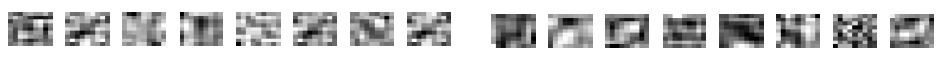

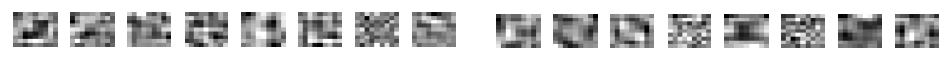

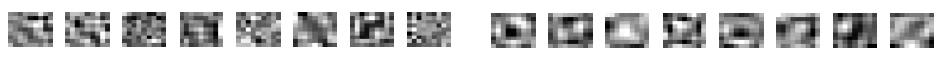

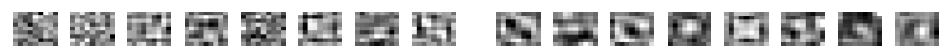

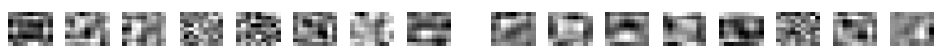

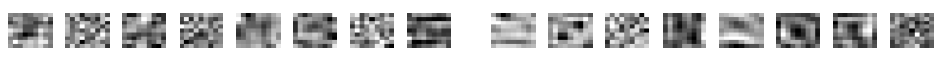

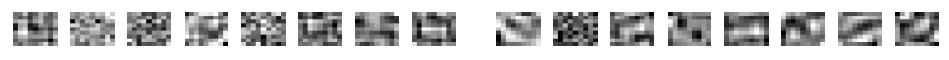

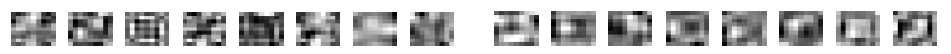
(a) SRCNN
(b) SRCNN-Pr

Figure 3: Visualization of filters in the first convolutional layers with magnification factor 3

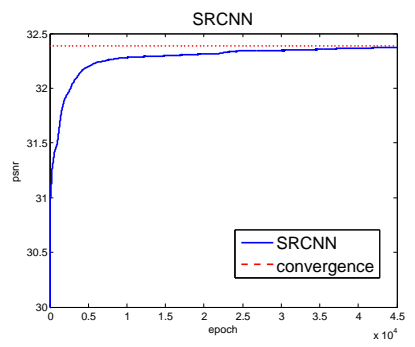

(a)

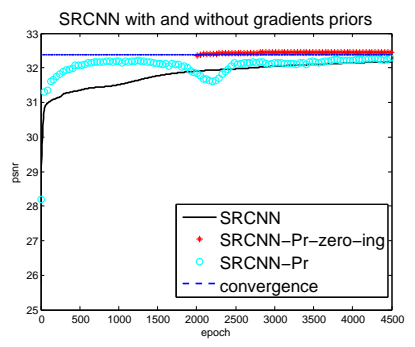

(b)

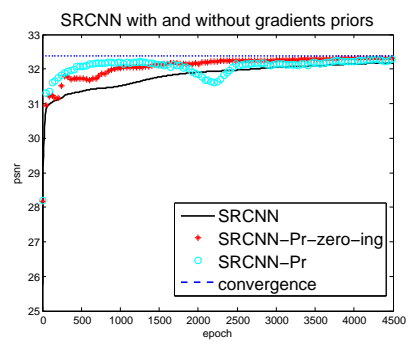

(c)

Figure 4: Comparison of test average psnr of Set 5 against training epochs between SRCNN with and without priors with a magnification factors 3: (a) denoted the test psnr of SRCNN,(b) and (c) explained the comparison for SRCNN with and without priors, (b) and (c) has applied different zeroing methods

accomplish super-resolution task with a magnification factor 2. As the first convolutional layer always captures the low-level information, we expected the first convolutional layer to be different across the scale. In the future, We can

further explore sharing the first convolutional layer and less parameters would be needed to trained.

We also incorporated image priors to further boost the performance of the our multi-task learning framework as Fig2, It achieved better results in both scores and visual observation, as well as faster training convergence. 


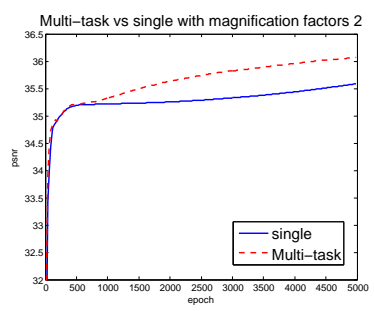

(a) $\bigotimes 2$

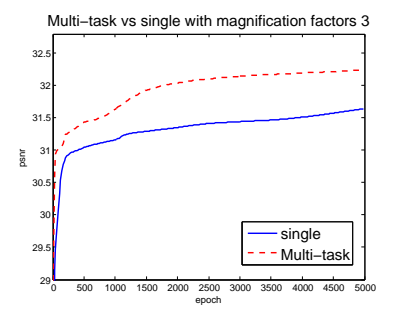

(b) $\bigotimes 3$

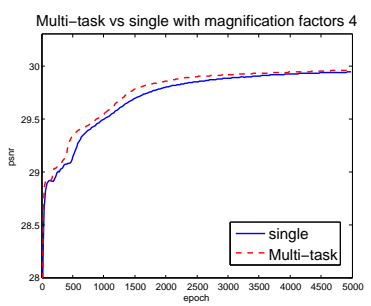

(c) $\otimes 4$

Figure 5: Comparison of test average psnr of Set5 against training epochs between multi-task learning and single task across different scales

\section{Experiments}

In this section, we reported a series of experiments to give intuitive visual demonstration and make the image quality assessments in comparison with state-of-the-art approaches.

In order to be compared with some state of art methods such as SRCNN [14], ANR [11, the same training set(91 images) and test sets were employed as in [14]. The Set5 [14](5 images) and Set14 [14](14 images) were used to evaluate the SR results. As 14, we only consider the luminance channel(in YCrCb colorspace) in our experiments. We use deep learning toolbox matconvnet [19] to re-implement Dong's work 14] with the same parameters and develop our approach. Specifically, we set three layers of convolutional filters for the SR part to be $[H, W, C, N]=9 \times 9 \times 1 \times 64,1 \times 1 \times 64 \times 32,5 \times 5 \times 32 \times 1$ respectively, which is exactly the same with Dong's work [14]. $H, W$ means receptive fields size of the filters and $C$ means the channel and $N$ means the filters number. The same learning rate for the first three convolutional layers $\left(10^{-4}, 10^{-4}, 10^{-5}\right)$ as Dong's work [14] were applied. For computational efficiency, only two fixed sobel filters were utilized to extracted the horizonal and vertical gradient information. We re-plotted the test convergence curve of average psnr values for Set5 against training epochs as our baseline(blue one). The dash lines in the figure of the paper denoted 'convergence' is the reported results after training for days in Dong's work [14]. It was roughly the same as the paper [14 in Fig 4(a). With 
more training epochs, the reconstruction results of Set5 is better. Dong [14] use number of back-propagations to measure the training time while we use epochs as Eq. 12 with batch size 128.

$$
\text { epochs }=\text { Num } m_{\text {back-propagations }} /(\text { batch_size } * \text { numofbatch })
$$

Prior modeling with two zeroing methods has been demonstrated in the experiment. One is simply set $\alpha=0$ in Eq. (6) after some training time as Fig $4(\mathrm{~b})$. while the other attempt is exponential decay of $\alpha$ as Eq. (8) with $d=0.997$ which gave out a smooth curve as Fig 4(c). As Fig 4(b)(c) shown, the black curve denoted the average psnr against training epochs when incorporating image gradients priors without zeroing methods. It quickly surpassed the baseline method at the very beginning with a large gaps. As its loss function had also taken the priors into consideration, the curve oscillated but still rose. When we use the zeroing methods, we can train the net with the priors at first then set $\alpha=0$ in Eq. 6. The red curve showed the behavior after we zeroing $\alpha$. Before zeroing $\alpha$, it had the same behavior as black curve. So we haven't plotted the red curve before zeroing $\alpha$ as it will cover the black curve. Zeroing $\alpha$ has boosted the performance thus the curve appeared to be discontinuous in Fig 4(b). The final loss function was the same as the reconstruction target and the curve with image gradients priors(red one) had quickly achieved a good psnr values and continued to show same behavior as baseline method trained with very long time. It proved the priors had guided the training towards a better direction at the beginning. What's more, incorporating priors was not just using more layers to fit the training samples. We found with priors the convergence process is much better than simply adding layers to fit the gradients. As observed in the experiment, little extra computational load has been added 125 as no parameter of feature extracting layers updated. As shown in Fig 4(a), when incorporating priors, with less than one-tenth of the training epochs $(4500$ epochs vs 45000 epochs) the algorithm has achieved better convergence than the dash convergence line reported by Dong 14 .

Although the learned mapping from LR to HR images has identical structure 


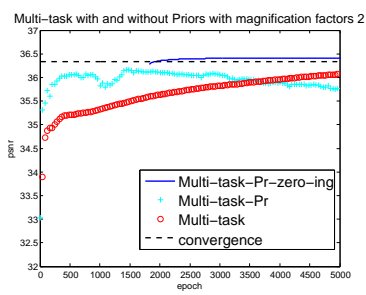

(a) $\otimes 2$

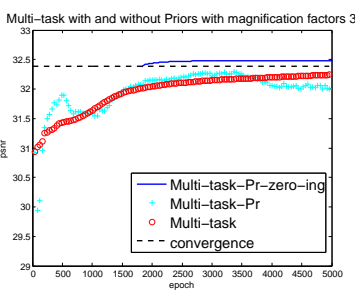

(b) $\otimes 3$

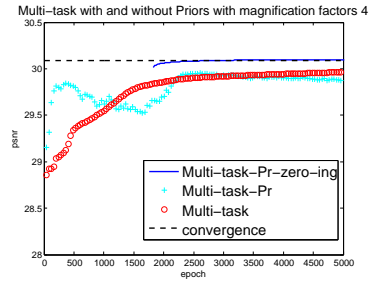

(c) $\otimes 4$

Figure 6: Comparison of test psnr of Set 5 against training epoch between multi-task learning with and without priors across different scales, Multi-task-Pr-zero-ing had the same behavior as Multi-task-Pr, zeroing alpha boosted the performance, so the curve appear to be discontinuous

as a Dong's SRCNN [14] convolutional neural network, our proposed method could achieve a better results and avoided the local minimum or over-fitting issues. We gave a visualized comparison of the learnt filters of the first convolutional layer between SRCNN(code from the author) and SRCNN-Pr. As can be seen from Fig 3 , the filters of our convolutional layers look more structured other than being randomize. More patterns have appeared such as the structure along diagonal direction. It validates that incorporating the image priors did help to ameliorate the illness nature and ambiguity problems. And It also demonstrated adding supervision information lead to discriminative learning of feature which did help to restore HR images.

Table 1: Comparison of Set5 with a magnification factors 3: P denotes PSNR scores(db), T denotes time(s)

\begin{tabular}{|c|c|c|c|c|c|c|c|c|c|c|c|c|c|c|}
\hline \multirow{2}{*}{$\begin{array}{c}\text { Set5 } \\
\text { images }\end{array}$} & \multicolumn{2}{|c|}{ Bicubic } & \multicolumn{2}{|c|}{$\mathrm{SC}$} & \multicolumn{2}{|c|}{ K-SVD } & \multicolumn{2}{|c|}{ NE+LLE } & \multicolumn{2}{|c|}{ ANR } & \multicolumn{2}{|c|}{ SRCNN } & \multicolumn{2}{|c|}{ SRCNN-Pr } \\
\hline & $\mathbf{P}$ & $\mathbf{T}$ & $\mathbf{P}$ & $\mathbf{T}$ & $\mathbf{P}$ & $\mathbf{T}$ & $\mathbf{P}$ & $\mathbf{T}$ & $\mathbf{P}$ & $\mathbf{T}$ & $\mathbf{P}$ & $T$ & $\mathbf{P}$ & $\mathbf{T}$ \\
\hline baby & 33.91 & - & 34.29 & 76.0 & 35.08 & 3.3 & 35.06 & 6.0 & 35.13 & 1.3 & 35.01 & 0.38 & 35.02 & 0.38 \\
\hline bird & 32.58 & - & 34.11 & 30.4 & 34.57 & 1.0 & 34.56 & 1.9 & 34.60 & 0.39 & 34.91 & 0.14 & 35.03 & 0.14 \\
\hline butterfly & 24.04 & - & 25.58 & 26.8 & 25.94 & 0.81 & 25.75 & 1.4 & 25.90 & 0.31 & 27.58 & 0.10 & 27.75 & 0.10 \\
\hline head & 32.88 & - & 33.17 & 21.3 & 33.56 & 1.0 & 33.60 & 1.7 & 33.63 & 0.35 & 33.55 & 0.13 & 33.56 & 0.13 \\
\hline woman & 28.56 & - & 29.94 & 25.1 & 30.37 & 1.0 & 30.22 & 1.9 & 30.33 & 0.37 & 30.92 & 0.13 & 30.93 & 0.13 \\
\hline average & 30.39 & - & 31.42 & 35.92 & 31.90 & 1.42 & 31.84 & 2.58 & 31.92 & 0.54 & 32.39 & 0.18 & 32.46 & 0.18 \\
\hline
\end{tabular}

Faster convergence and better restoration can be analyzed from following aspect: first, modeling image restoration with the image gradients consistency measure acted as a type of regularization or as a guided supervised training 


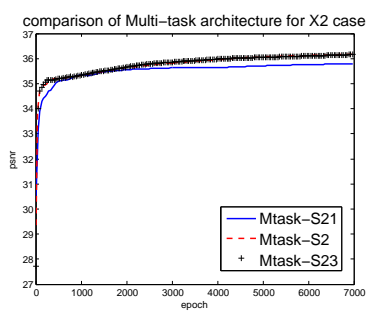

(a) $\bigotimes 2$

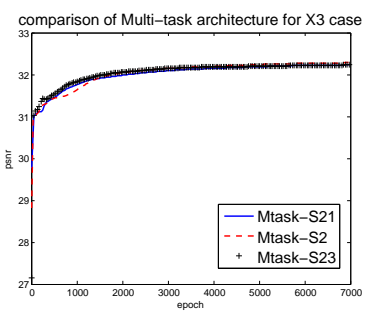

(b) $\otimes 3$

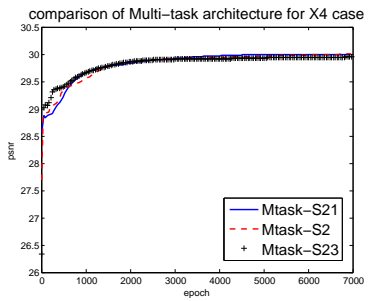

(c) $\otimes 4$

Figure 7: Comparison of test average psnr of Set5 against training epochs between different multi-task learning CNN archtecture across different scales, Mtask-S21, Mtask-S2 and Mtask-S23 denotes sharing the weights of (1st, 2nd),(2nd) and (2nd, 3rd) convolutional layers respectively.

information. Second, a richer output implies higher error to be propagated from the top layer which is partly equivalent to increasing the learning rate of the CNN. However, modeling the image statistical issue is the key to improve the situation. Simply increasing the learning rate of the net will explode the error propagation and damage the convergence. the consistency measure can also be interpreted as a good initialization method by utilizing the priors like image gradients.

As Fig 5 learning a mapping of different magnification factors simultaneously has also regulated the training efficiently and effectively. Better rising and converging of the curve has been observed. What's more, at one time, we can get several mappings from LR to HR images of different magnification factors. It is interesting to find in $\bigotimes 4$ case, boost is not that apparent. The situation is that the reported psnr in Dong [14] is $30.09 \mathrm{~dB}$, which is rather approximate by the curve. It means little boost will need a huge extra training.

In Dong's work [14, three convolutional layers were divided into three phrase respectively, which is patch extraction and representation phrase, non-linear mapping phrase and reconstruction phrase. The first layer captures the low level information which need more variations among different upscaling tasks. It is reasonable to share the nonlinear mapping across tasks to regulate each other. We further explore whether the reconstruction layer should be further 
shared. In 7. the average test psnr of Set5 with different multitask learning CNN structures were compared. Mtask-S21, Mtask-S2 and Mtask-S23 denotes multitask CNN structure which shared the weights of (1st, 2nd),(2nd) and (2nd, 3rd) convolutional layers respectively. Weight sharing 1st and 2nd convolutional layers has damaged the performance as the training iterated to extract the discriminative features especially in $\bigotimes 2$ case. In $\bigotimes 2$ case, input images contains more texture details than other cases, thus the filters of first layer should be different. Curve slightly dropped as weights of 3rd convolutional layer were further shared. Reconstruction phrase may prefer different filters to restore texture and structures for each upscaling cases. However, fewer parameters would be needed to be stored in the memory which was a compromise between quality and storage cost.

Table 2: Comparison of Set14 with a magnification factors 3: P denotes PSNR scores(db), T denotes time(s)

\begin{tabular}{|c|c|c|c|c|c|c|c|c|c|c|c|c|c|c|}
\hline Set14 & Bict & & & & $\mathrm{K}-\mathrm{s}$ & & $\mathrm{NE}-$ & & & & SRC & & SRCI & $\mathrm{J}-\mathrm{Pr}$ \\
\hline images & $\mathbf{P}$ & $T$ & $\mathbf{P}$ & $T$ & $\mathbf{P}$ & $\mathbf{T}$ & $\mathbf{P}$ & $T$ & $\mathbf{P}$ & $\mathbf{T}$ & $\mathbf{P}$ & $T$ & $P$ & $T$ \\
\hline average & 27.54 & - & 28.31 & 84.88 & 28.67 & 2.95 & 28.60 & 5.35 & 28.65 & 0.97 & 29.00 & 0.39 & 29.06 & 0.39 \\
\hline
\end{tabular}

In table 1 and 3 , SRCNN-Pr and SRCNN-Multitask-Pr achieve average psnr as $32.46 \mathrm{db}$ and $32.48 \mathrm{db}$ respectively on Set5 with upscaling factor 3 . Although the boost from SRCNN-Pr to SRCNN-Multitask-Pr is small, it did regulate the training and achieve multiple upscaling models at one time.

As Fig 6, incorporating the image priors has also accelerated the training effectiveness of multi-task and given better results. Some pictures were given for visual comparison in Fig 8, Our results were visually more pleasant, sharper and more natural in texture-rich regions in much less training time. For example, abdomen of the bird was more natural by our methods than SRCNN 14] while our's appeared more clear and sharper than ANR [11. The restored eyelid of boy also is more clear by our methods. It proves incorporating image priors in this way generally accelerates the training and better handles the image edge and texture areas. 
Table 3: Comparison of average psnr across scales

\begin{tabular}{|l|c|c|c|c|}
\hline \multicolumn{5}{|c|}{ Average psnr of Set5 } \\
\hline & SRCNN-Multitask-Pr & SRCNN & ANR & KK \\
\hline$\otimes 2$ & $\mathbf{3 6 . 4 1}$ & 36.34 & 35.83 & 36.20 \\
\hline$\otimes 3$ & $\mathbf{3 2 . 4 8}$ & 32.39 & 31.92 & 32.28 \\
\hline$\otimes 4$ & $\mathbf{3 0 . 1}$ & 30.09 & 29.69 & 30.03 \\
\hline \multicolumn{5}{|c|}{ Average psnr of Set 14} \\
\hline$\otimes 2$ & $\mathbf{3 2 . 2 2}$ & 32.18 & 31.80 & 32.11 \\
\hline$\otimes 3$ & $\mathbf{2 9 . 0 5}$ & 29.00 & 28.65 & 28.94 \\
\hline$\otimes 4$ & $\mathbf{2 7 . 2 5}$ & 27.20 & 26.85 & 27.14 \\
\hline
\end{tabular}

\section{Conclusion}

In this paper, we present a multi-task deep convolutional neural network framework with image priors modeling and multi-scale information regulating. By incorporating image priors, the proposed framework could effectively improve training convergence speed(less than one-tenth training epochs) and pay special attention to the edges and texture regions. The proposed framework simultaneously trained SR deep convolutional neural network of multiple magnification factors and exploited the structure recurrence across different scales. It achieved visually and objectively better results against state of art methods. The future work mainly includes investigating the deep convolutional neural network in the same fashion with different priors and different CNN structures to exploit the structure recurrence across different scales for image super-resolution problem.

\section{Acknowledgment}

This work is partially supported by the Open Projects Program of National Laboratory of Pattern Recognition, and the National Science Foundation of China under Grant No.61473219. We are very appreciate for Chao Dong's great

help and thoughtful advice.

[1] R. Keys, Cubic convolution interpolation for digital image processing, Acoustics, Speech and Signal Processing, IEEE Transactions on 29 (6) (1981) $1153-1160$. 
[2] M. Irani, S. Peleg, Motion analysis for image enhancement: Resolution, occlusion, and transparency, Journal of Visual Communication and Image Representation 4 (4) (1993) 324-335.

[3] H. A. Aly, E. Dubois, Image up-sampling using total-variation regularization with a new observation model, Image Processing, IEEE Transactions on 14 (10) (2005) 1647-1659.

[4] W. T. Freeman, E. C. Pasztor, O. T. Carmichael, Learning low-level vision, International journal of computer vision 40 (1) (2000) 25-47.

[5] J. Yang, J. Wright, T. Huang, Y. Ma, Image super-resolution as sparse representation of raw image patches, in: Computer Vision and Pattern Recognition, 2008. CVPR 2008. IEEE Conference on, IEEE, 2008, pp. 1-8.

[6] D. Glasner, S. Bagon, M. Irani, Super-resolution from a single image, in: Computer Vision, 2009 IEEE 12th International Conference on, IEEE, 2009, pp. 349-356.

[7] J. Yang, Z. Lin, S. Cohen, Fast image super-resolution based on in-place example regression, in: Computer Vision and Pattern Recognition (CVPR), 2013 IEEE Conference on, IEEE, 2013, pp. 1059-1066.

[8] J. Yang, J. Wright, T. S. Huang, Y. Ma, Image super-resolution via sparse representation, Image Processing, IEEE Transactions on 19 (11) (2010) $2861-2873$.

[9] H. Zhang, J. Yang, Y. Zhang, T. S. Huang, Multi-scale non-local kernel regression for super resolution, in: Image Processing (ICIP), 2011 18th IEEE International Conference on, IEEE, 2011, pp. 1353-1356.

[10] T. Michaeli, M. Irani, Nonparametric blind super-resolution, in: Computer Vision (ICCV), 2013 IEEE International Conference on, IEEE, 2013, pp. 945-952. 
[13] C.-Y. Yang, C. Ma, M.-H. Yang, Single-image super-resolution: A benchmark, in: Computer Vision-ECCV 2014, Springer, 2014, pp. 372-386.

[14] C. Dong, C. C. Loy, K. He, X. Tang, Learning a deep convolutional network for image super-resolution, in: Computer Vision-ECCV 2014, Springer, 245

[11] R. Timofte, V. De, L. V. Gool, Anchored neighborhood regression for fast example-based super-resolution, in: Computer Vision (ICCV), 2013 IEEE International Conference on, IEEE, 2013, pp. 1920-1927.

[12] C.-Y. Yang, M.-H. Yang, Fast direct super-resolution by simple functions, in: Computer Vision (ICCV), 2013 IEEE International Conference on, IEEE, 2013, pp. 561-568. 2014, pp. 184-199.

[15] C. Dong, C. C. Loy, K. He, X. Tang, Image super-resolution using deep convolutional networks, arXiv preprint arXiv:1501.00092.

[16] C.-Y. Lee, S. Xie, P. Gallagher, Z. Zhang, Z. Tu, Deeply-supervised nets, arXiv preprint arXiv:1409.5185.

[17] I. Sobel, History and definition of the sobel operator.

[18] A. Krizhevsky, I. Sutskever, G. E. Hinton, Imagenet classification with deep convolutional neural networks, in: Advances in neural information processing systems, 2012, pp. 1097-1105.

[19] A. Vedaldi, K. Lenc, Matconvnet - convolutional neural networks for matlab, CoRR abs/1412.4564. 

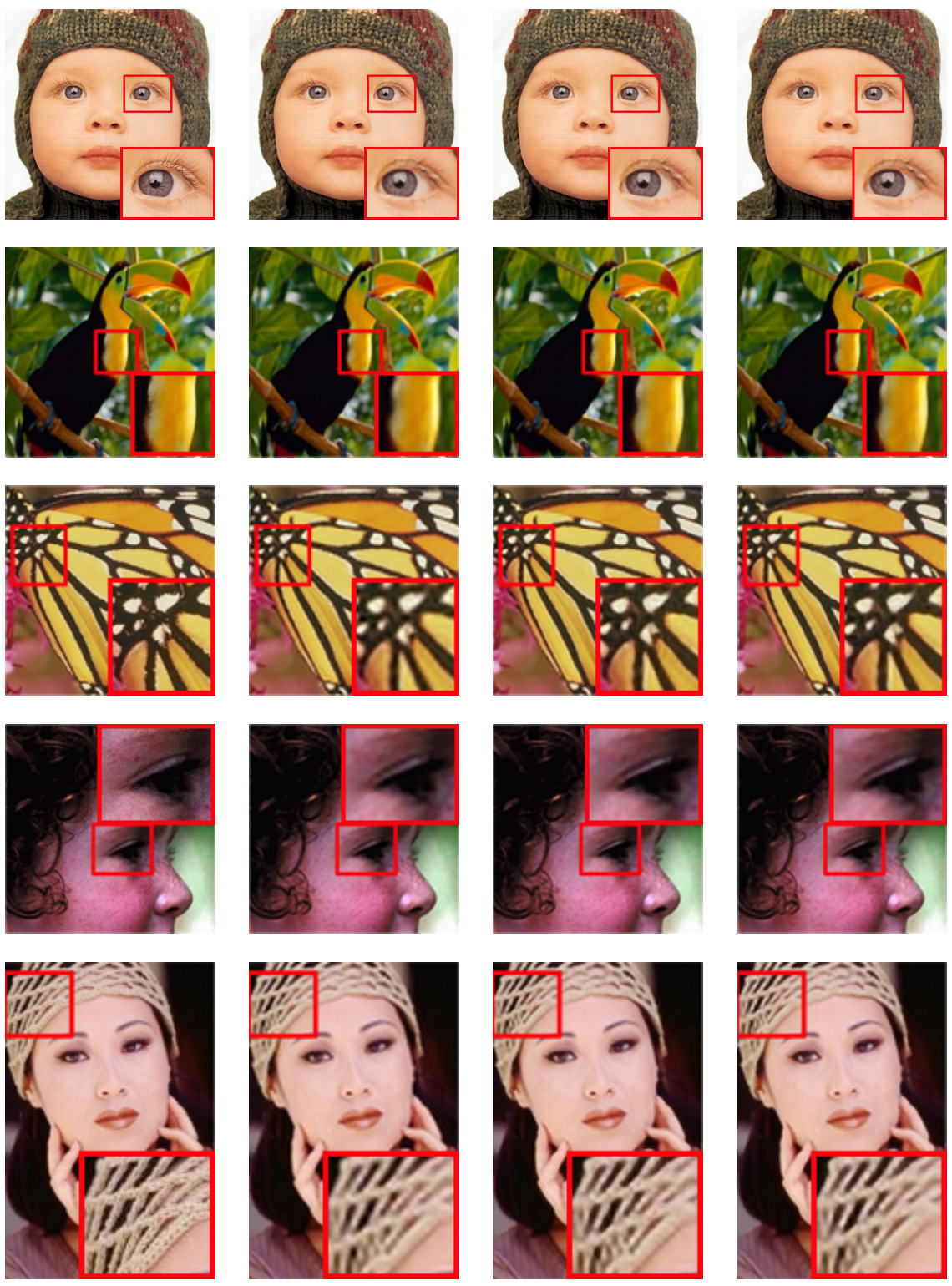

(a) groundtruth

(b) ANR 11]

(c) SRCNN

(d)SRCNN-Pr

Figure 8: Visual comparison of different SR method with a magnification factors 3 
Yudong Liang received the B.S. degree in Electronic Engineering from Xi’an Jiaotong University, Xi’an, China, in 2010. He is currently pursuing the Ph.D. degree in Institute of Artificial Intelligence and Robotics at Xi'an Jiaotong University. His research interests include machine Learning and computer Vision, with a focus on image super-resolution, image quality assessment and deep learning.

Jinjun Wang received the B.E. and M.E. degrees from the Huazhong University of Science and Technology, China, in 2000 and 2003, respectively. He received the Ph.D. degree from Nanyang Technological University, Singapore, in 2006. From 2006 to 2009, he was with NEC Laboratories America, Inc., as a Research Scientist, and Epson Research and Development, Inc., as a Senior Research Scientist, from 2010 to 2013. He is currently a Professor with Xi'an Jiaotong University. His research interests include pattern classification, image/video enhancement and editing, content-based image/video annotation and retrieval, semantic event detection, etc.

Sanping Zhou received the M.E. degree from Northwestern Polytechnical University, Xi'an, China, in 2015. He is currently pursuing the Ph.D. degree in Institute of Artificial Intelligence and Robotics at Xi'an Jiaotong University. His research interests include medical image segmentation, deep learning, person re-identification, and visual tracking.

Yihong Gong received the B.S., M.S., and Ph.D. degrees in electrical engineering from The University of Tokyo, Japan, in 1987, 1989, and 1992, respectively. In 1992, he joined Nanyang Technological University, Singapore, as an Assistant Professor with the School of Electrical and ElectronicEngineering. From 1996 to 1998, he was a Project Scientist with the Robotics Institute,Carnegie Mellon University, USA. Since 1999, he has been with the Silicon Valley branch, NEC Labs America, as a Group Leader, the Department Head, and the Branch Manager. In 2012, he joined Xi'an Jiaotong University, China, as a Distinguished Professor. His research interests include image and video analysis, multimedia database systems, and machine learning.

Nanning Zheng graduated from the Department of Electrical Engineering, Xi'an Jiaotong University, Xi'an, China, in 1975, and received the M.S. degree in information and control engineering from Xi'an Jiaotong University in 1981 and the Ph.D. degree in electrical engineering from Keio University, Yokohama, Japan, in 1985. He jointed Xi'an Jiaotong University in 1975, and he is currently a Professor and the Director of the Institute of Artificial Intelligence and Robotics, Xi'an Jiaotong University. His research interests include computer vision, pattern recognition and image processing, and hardware implementation of intelligent systems. Dr. Zheng became a member of the Chinese Academy of Engineering in 1999, and he is the Chinese Representative on the Governing Board of the International Association for Pattern Recognition. He also serves as the President of Chinese Association of Automation. 


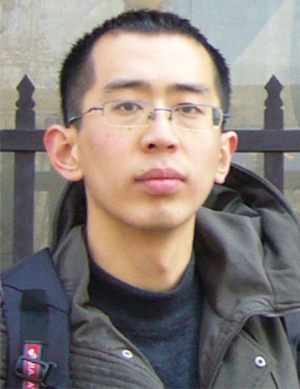




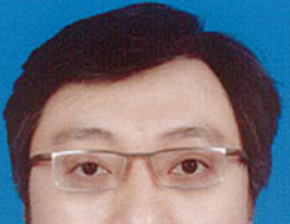




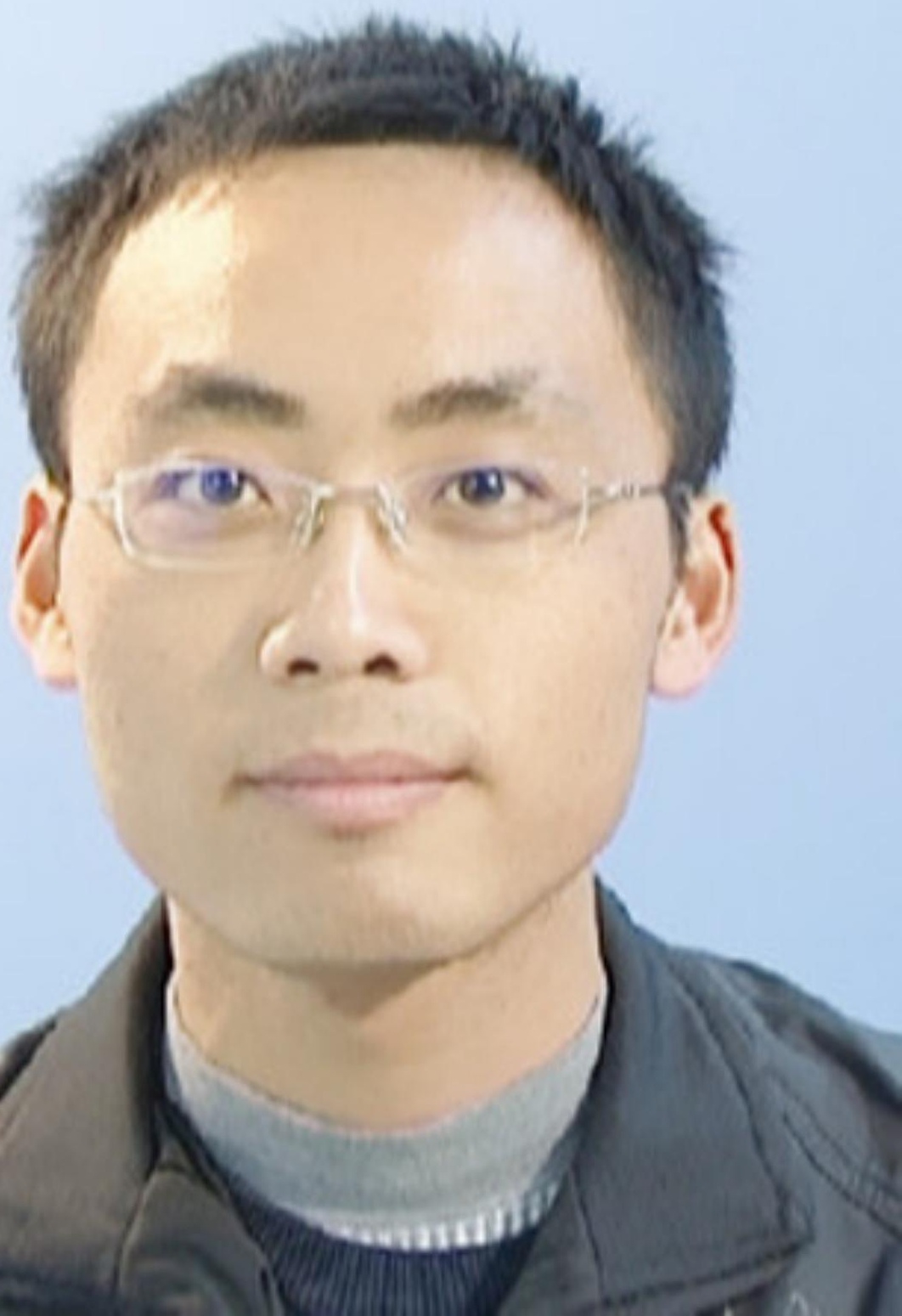




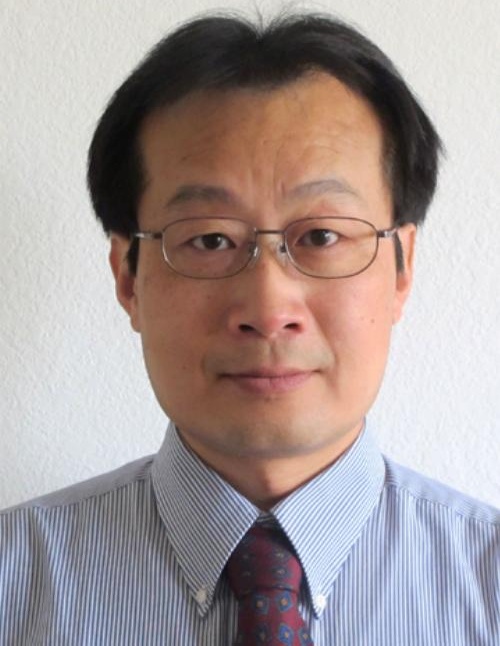



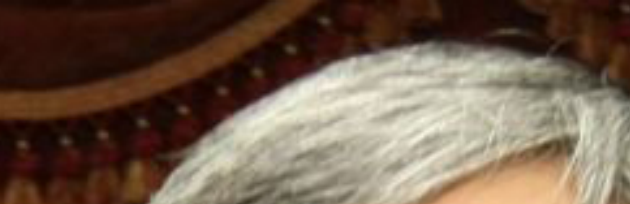